\title{
Hervé Guibert : de la quête identitaire au plaisir du corps
}

\author{
Anca Porumb \\ Université de Babes-Bolyai
}

Problème philosophique, métaphysique et anthropologique, le corps humain a suscité de nombreuses interrogations. Le regard le perçoit avec fascination et peur, car le corps révèle le caractère unique de l'être. C'est par lui que l'homme réussit à se découvrir et à se connaître, en refusant le corps de l'autre, vu par l'autre. Analysant L'Être et le Néant, Claude Bruaire affirme :

[...] si, au moyen de la négation du corps d'autrui, comme expression de son être singulier, en vertu de l'identité de l'âme et du concept, l'attention aux paroles, au langage d'autrui, à son corps parlant, était dénaturée et supprimée, c'est maintenant, au moyen de l'âme et de la liberté singulière, que l'expressivité 
du corps d'autrui est ruinée pour la connaissance en première personne. (1968, p. 113)

Bruaire distingue le corps expressif et le corps comme action. Nous pourrions transposer cette philosophie du corps expressif, envisagé comme source de dualité, pour l'interprétation de l'œuvre de Guibert, pour qui la découverte de son propre corps représente l'effort de se construire une nouvelle identité. L'écrivain se contemple tel Narcisse et prend conscience de son double. L'angoisse le saisit devant l'image de l'autre moi. Il n'y a pas de sentiments d'amour face à cette réflexion; il n'y a pas de désir comme dans le mythe. Se regarder dans le miroir signifie, pour les protagonistes des romans d'Hervé Guibert, revenir à la réalité cruelle de la maladie. Le miroir est un motif récurrent et s'avère indispensable dans la tentative de se (re)connaître. La fonction attribuée au miroir par Jackie Pigeaud correspond assez à la démarche de Guibert :

Le miroir est donc l'instrument de la connaissance de soi. On ne peut se connaître que médiatement. Le miroir permet l'analogie, sans laquelle on ne se peut connaître; il est l'instrument de la scission. Se connaître, c'est se voir deux, se comparer entre deux, et s'apprécier. (2009, p. 20)

Qu'il y ait besoin d'un médiateur, nous ne le nions pas. Mais chez les écrivains qui ont reconnu leur condition homosexuelle et la maladie, la rupture de leur moi pourrait être mesurée en secondes. Le miroir les aide à se percevoir tels quels, bien qu'il leur arrive parfois de se dédoubler.

La manière avec laquelle Guibert envisage le motif du miroir nous amène à analyser l'image du corps comme source des maux et des changements anatomiques, les esthétiques du beau et du laid s'entrecroisant dans les romans de l'auteur. 


\section{Le corps en décrépitude}

L'année 1990 plonge le monde littéraire dans la confusion avec le roman Á l'ami qui ne m'a pas sauvé la vie. Guibert n'est pas un auteur réaliste qui se contente d'écrire sur le sida. Il y a chez lui un hyper-réalisme qui va plutôt vers le naturalisme. En d'autres termes, l'œuvre guibertienne pourrait se définir comme choquante par la richesse des détails cruels sur le corps humain en dégradation, par le penchant de l'écrivain pour l'observation de chaque étape de sa maladie. Guibert ne se veut pas le représentant de la communauté homosexuelle. Il veut tout simplement faire une analyse de soi à soi et de soi aux autres. Dans ses deux romans les plus connus, Á l'ami qui ne m'a pas sauvé la vie et Le Protocole compassionnel, il ne ménage personne. La sincérité avec laquelle il nourrit les controverses. Peut-être l'a-t-il voulu, parce que le début du roman n'a besoin d'aucun commentaire : "J'ai eu le sida pendant trois mois. Plus exactement, j'ai cru pendant trois mois que j'étais condamné par cette maladie mortelle qu'on appelle le sida. » (1990, p. 9)

Cet incipit est ambigu, mais l'auteur continue à nous surprendre dans les lignes suivantes : «Or je ne me faisais pas d'idées, j'étais réellement atteint, le test qui s'était avéré positif en témoignait, ainsi que des analyses qui avaient démontré que mon sang amorçait un processus de faillite. » (1990, p. 9)

Est-il ou n'est-il pas atteint de la maladie ? S'il joue avec nous dans les premières pages, Guibert ne plaisantera plus par la suite en parlant du sida et de lui-même. Il a le sida, sans aucun doute. Le titre du livre, qui assure l'unité du roman, en est la preuve. Bien que son œuvre ressemble à un traité de médecine, la trame est née de l'existence d'un personnage, Bill. 
C'est l'ami américain de Guibert qui refuse de lui procurer le médicament qui pourrait le guérir. Maintenant, l'auteur sera l'acteur et le metteur en scène du scénario de la mort qui approche. Il choisit ce qui est le plus dur : s'écrire en tant que malade et écrire les expériences sinistres par lesquelles le sida l'oblige à passer, en essayant de trouver une explication ou, plutôt, une définition au mal qui le ronge :

[...] le sida n'est pas vraiment une maladie, ça simplifie les choses de dire que c'en est une, c'est un état de faiblesse et d'abandon qui ouvre la cage de la bête qu'on avait en soi, à qui je suis contraint de donner pleins pouvoirs pour qu'elle me dévore, à qui je laisse faire sur mon corps vivant ce qu'elle s'apprêtait à faire sur mon cadavre pour le désintégrer. (1990, p. 17)

Faisant du sida une question personnelle, individuelle, Guibert n'a que son propre corps sur lequel se pencher. C'est tout ce qui lui reste, et l'auteur en fait une véritable obsession. Plus qu'une obsession, le corps est la seule liaison avec la vie. À travers l'analyse de son corps malade, Guibert peut se donner chaque matin la certitude, en se regardant dans le miroir, qu'il est encore vivant. Toute maladie, et particulièrement le sida, agit d'abord sur le physique et, ensuite, sur le psychisme du malade. Les changements évidents que le corps subit obligent la personne malade à se percevoir différemment à chaque étape de la maladie :

Reflet et miroir qui se donne à voir tout en donnant à voir, lieu de l'être et du paraître où se repèrent les usages sociaux et où se lisent les marques du temps, le corps est tout à la fois celui du travail, du plaisir et du plaire et celui de la maladie, de la douleur et de la mort. Que lit-on sur le corps malade et comment lit-on le corps malade? Mais aussi que pouvons-nous et que savons-nous en dire? Il y a toujours, en effet, un indicible du corps; un silence ou des cris qui ne se peuvent articuler. Nous sommes toujours dominés et muets face aux 
cataclysmes de notre corps. Mais, pour cette raison entre autres, le regard que nous portons sur lui, s'il veut parvenir à connaître et à dire, doit prendre de la distance, choisir et sérier les manifestations du corps, les convertir en signes. La lecture du corps est toujours une construction : du corps lui-même et de ses rapports à la maladie. (Herzlich et Pierret, p. 101)

Dominé et muet face aux cataclysmes de son corps, tel est bien Guibert, qui se trouve impuissant face à la maladie destructrice. Pourtant, cette attitude change vite, parce que l'écrivain décide de ne pas rester muet, de dominer le sida par l'écriture. Aucun autre avant lui n'a décrit avec autant de détails médicaux les examens cliniques que son corps accepte. Le narrateur/patient n'est plus préoccupé par l'aspect physique comme source de plaisir. Le sida menace trop sa vie pour qu'il ait encore la force de penser aux relations qui sont à l'origine de son mal. La seule fonction du corps est désormais de faire face au travail, malgré l'affaiblissement, les difficultés à écrire dues aux douleurs atroces qui annoncent la fin.

Guibert se détache progressivement du miroir et rentre dans un processus qui consiste à trouver une signification aux manifestations de la maladie. Pour pouvoir décrire le sida, l'écrivain est obligé de prendre de la distance et de parler de soi en se reflétant dans le miroir ou la caméra. La lecture du corps que Guibert choisit de mener naît du désir de se retrouver dans chaque personne qu'il découvre en se regardant ou en se filmant.

Il surprend d'abord la dégradation physique par le truchement des amis. Comme un préambule à ce qui suivra, l'auteur fait l'apprentissage de la maladie et de la fin inévitable en racontant les expériences des autres. Les changements de leurs corps ainsi que les modifications psychiques le 
bouleversent comme s'il les vivait lui-même. De plus, l'auteur se rend compte de la menace que représente le sida : la perte de l'identité.

En associant la dégradation du corps à la perte de l'identité, Guibert aborde l'aspect social du sida sans trop y insister. Plus attentif que jamais aux transformations physiques causées par la maladie, l'auteur nous met en présence d'un véritable traité de médecine. Sa démarche est explicable. Sa souffrance est si grande qu'il ne sent plus son corps lui appartenir. Celui-ci s'est détaché de l'âme et agit séparément. L'équilibre est rompu et le corps sera toujours un pas en avant, en disposant à son gré de la vie de l'écrivain. Devenus déjà personnages principaux du livre, le sida et, implicitement, le corps sont trop présents et mènent à l'insupportable :

Puisque le corps se retrouve frustré, par cette annonce de la malformation rénale bénigne puis cette théorie de la spasmophilie, dépossédé momentanément de ses capacités de souffrance, sans doute avide il se remit à forer en lui, au plus profond, aveuglément, à tâtons. Je ne faisais pas de crises d'épilepsie, mais j'étais capable à chaque instant de me tordre littéralement de douleur. (Guibert, 1991, p. 45)

La douleur organique qui se glisse sournoisement dans le corps encore vivant et sensible aux sensations est transmise aussi aux lecteurs. Il est impossible de ne pas souffrir en même temps que le narrateur, car les mots ne ménagent pas la réalité. Le mot " souffrance » perd de son intensité. Il y a quelque chose qui va au-delà de la capacité à endurer une maladie épuisante. Le malade ne se perçoit plus comme un tout qu'il formerait avec son corps. Guibert parle de son corps comme d'une entité à part. Ce n'est plus sa frustration venant de l'impossibilité de bouger. C'est la frustration du corps démuni du pouvoir de 
lutter contre l'intrus qui le ronge de l'intérieur. Guibert est luimême l'objet de son analyse, auteur et narrateur à la fois. Pour le narrateur, Hervé, la mort est une provocation. Il mène une lutte continuelle avec le sida, qui devient maître de son corps. Celui-ci ne répond plus à ses désirs et il n'a qu'un seul souci, celui d'avoir le temps et la force de raconter son histoire jusqu'à la fin :

Le livre lutte avec la fatigue qui se crée de la lutte du corps contre les assauts du virus. Je n'ai que quatre heures de validité par jour, une fois que j'ai remonté les stores immenses de la verrière, qui sont le potentiomètre de mon souffle déclinant, pour retrouver la lumière du jour et me remettre au travail. [...] Ce livre qui raconte ma fatigue me la fait oublier [...]. (Guibert, 1990, p. 66-67)

N'ayant plus la force de continuer le combat avec le sida, Guibert donne la parole au livre après avoir laissé son corps décider à sa place. L'écrivain/narrateur revient pourtant au « je » et retrouve son souffle à travers l'écriture. Le corps n'a plus droit de vie et de mort sur lui. Sa force s'affaiblit devant celle des mots qui racontent la maladie. Guibert met toute sa confiance dans l'acte d'écriture pour avoir le courage de franchir les obstacles auxquels le sida le fait se heurter. La maladie des autres l'a préparé à sa maladie et la découverte de la dégradation des autres a servi à la découverte de son propre corps :

J'ai vu des gens dans un état terrible, ce matin à Rothschild. Vraiment de jeunes cadavres aux yeux de braise, comme sur des affiches de films d'horreur où des morts ressortent de leurs tombes et font quelques pas en vacillant. J'ai l'impression qu'ils sont en plus mauvais état que moi, mais peut-être que personne ne se voit lui-même tel qu'il est, peut-être qu'il subsiste un tel narcissisme fût-ce dans la personne la plus 
abimée qu'elle n'est jamais capable que d'estimer les ravages de l'autre. (Guibert, 1991, p. 46)

La perception du temps change avec le changement du corps :

j'étais désormais incapable de faire aucun de ces gestes sinon au prix de gesticulations et d'efforts grimaçants, un corps de vieillard avait pris possession de mon corps d'homme de trente-cinq ans, il était probable que dans la déperdition de mes forces j'avais largement dépassé mon père qui vient d'en avoir soixante-dix, j'ai quatre-vingt-quinze ans, comme ma tante Suzanne qui est impotente (Guibert, 1991, p. 10).

La décrépitude corporelle provoquée par le sida plonge le malade dans la confusion. Les efforts pour se saisir s'avèrent un échec. Plus le narrateur veut se voir réapparaître tel qu'il était autrefois, plus le corps disparaît. Le sida suit sa voie et le transforme en un vieillard qui a perdu tout contrôle sur ses mouvements.

Le temps, qui passe trop vite, peut être interprété de deux manières. D'une part, la maladie détériore la condition physique de l'homme à tel point que celui-ci ne se reconnaît plus, malgré le désir de regagner son identité. D'autre part, le narrateur malade est conscient du fait que le sida ne lui permet plus de vieillir :

Le sida m'a fait accomplir un voyage dans le temps, comme dans les contes que je lisais quand j'étais enfant. Par l'état de mon corps, décharné et affaibli comme celui d'un vieillard, je me suis projeté, sans que le monde bouge si vite autour de moi, en l'an 2050. (1991, p. 111)

Le voyage imaginaire que Guibert met en scène dans son livre s'inscrit encore dans la fiction volontaire existant dans l'œuvre de l'écrivain. Guibert ne renonce pas au dédoublement continu de sa personne, où le « je » réel et le « je » fictionnel ne cessent 
de donner aux lecteurs l'impression de deux «moi » qui ne se rencontrent jamais.

Dans ce drame de la quête identitaire, Guibert trouve l'aspect positif de la maladie. La description qu'il en donne révèle son sang-froid et aussi la maturité de sa pensée. Les associations inédites des mots démontrent à quel point le malheur peut changer la vision de la vie. L'auteur se considère déjà comme le partenaire du sida, qui lui ouvre d'autres perspectives dans la façon de voir le monde et son existence :

Et c'est vrai que je découvrais quelque chose de suave et d'ébloui dans son atrocité, c'était certes une maladie inexorable, mais elle n'était pas foudroyante, c'était une maladie à paliers, un très long escalier qui menait assurément à la mort mais dont chaque marche représentait un apprentissage sans pareil, c'était une maladie qui donnait le temps de mourir, et qui donnait à la mort le temps de vivre, le temps de découvrir le temps et de découvrir enfin la vie [...]. (1990, p. 181)

Cet extrait est la quintessence de l'esthétique des romans de Guibert, qui fait de la mort la raison de vivre et découvre dans la vie la périssabilité de l'homme.

\section{Le « je » sidéen}

Le repli sur soi-même est déjà évident, et c'est à travers la famille que Guibert observe et écrit en tant qu'homosexuel. C'est une première étape du «je » qui s'analyse et qui veut renoncer à lui-même pour s'en construire un autre, nouveau et totalement coupé du passé. L'auteur passe du « je » homosexuel $\mathrm{au}$ « je » malade, obligé de trouver un moyen pour faire face à la mort, de se fixer une attitude devant cette mort qui approche. La maladie et la mort envahissent l'univers guibertien et mettent l'être humain à l'écart des autres. C'est à elles de faire la loi dans ses livres et d'organiser l'écriture. La mort, même si elle 
est racontée à la première personne, n'épargne pas les autres, et ici apparaît le caractère existentialiste de l'œuvre de Guibert. L'écrivain s'engage à dire ouvertement les tragédies auxquelles l'homme est confronté pendant sa vie. Ses romans, Á l'ami qui ne m'a pas sauvé la vie et Le Protocole compassionnel, sont un jeu entre l'individuel (le « je ») et l'universel (le « nous»), parce que la mort est universelle et que la maladie peut survenir à tout moment et à tout être humain.

Guibert choisit d'écrire le sida dans les moindres détails. L'auteur écrit son sida sans pudeur, en glissant vers la pornographie. Son désir est de « coucher le moi sur le papier» (Boulé, p. 285) dans sa permanente agonie entre la joie - parce que «le sida est une maladie merveilleuse » (Guibert, 1990, p. 18) - et la peur - parce qu'« on va tous crever de cette maladie » (1990, p.117). Le sida déchire le personnage/narrateur physiquement aussi bien que moralement. La présence de la maladie dans le corps agit comme facteur destructeur, à l'extérieur d'abord. La difficulté de se reconnaître est le premier signe que le malade perçoit comme la perte de l'identité. Il n'est plus sûr de son propre « je » et a besoin de se mirer souvent pour se convaincre de son existence.

Le drame de la maladie réside pourtant dans la dégradation de l'âme plus que dans la dégradation physique. Le malade vit à la fois avec le sentiment de la mort imminente et avec l'espoir d'entendre un jour parler de la découverte du médicament salvateur. La lutte psychologique est dure, comme le raconte l'écrivain tout au long de ses livres. Il est partagé entre la vie et la mort. 
Après s'être habitué à la vue d'un corps délabré, Guibert a encore la force de croire à la guérison. L'auteur s'accroche à l'amitié de Bill, l'Américain, qui le bouleverse et lui donne l'espoir de vaincre le sida grâce au vaccin que l'Amérique vient de réaliser. Arrivé en France pour apporter la bonne nouvelle, Bill monopolise le dialogue, dont l'importance est capitale pour Guibert, qui le situe avec une grande précision dans le temps, le 18 mars 1988 :

Bill est dans un état d'excitation indescriptible, qui va emporter avec lui notre dîner, et monopoliser toutes nos conversations : il nous annonce tout de go qu'on vient de mettre au point en Amérique un vaccin efficace contre le sida, pas vraiment un vaccin, pour être exact, puisque en principe un vaccin est préventif, alors disons un vaccin curatif, obtenu à partir du virus HIV et administré à des séropositifs non symptomatiques, appelés initialement les "porteurs sains » jusqu'à ce qu'on remette en cause le côté sain d'un homme contaminé par le sida, de façon à bloquer la virulence, à empêcher le virus de mettre en branle son processus de destruction [...]. (Guibert, 1990, p. 173)

Animé par les mots de Bill, le malade pense redevenir la personne de l'époque où le sida n'avait changé que le corps. Le « je » du passé revient, encouragé par la découverte du remède tant recherché. Son psychisme s'efforce d'accepter les extrêmes : croire entièrement à la vie et à la médecine ou se laisser porter par le destin clairement représenté par les symptômes de la maladie :

J'ai peur d'être blême, ou soudain trop rouge, j'ai peur de me trahir, et pour me défaire une bonne fois de cette peur je lance à Bill avec l'ironie : "Alors tu vas nous sauver, tous ici autant que nous sommes? - Ne dis pas de bêtises, me rétorque Bill gêné dans le développement de son récit [...]. (1990, p. 174) 
L'ironie dans la voix trahit la méfiance de Guibert dans l'attitude trop hautaine de son ami américain. Le dévoilement du secret à celui qui n'inspire pas de confiance lui confirme l'hypocrisie du système médical, soit-il français ou américain. Et ce qui blesse le plus, c'est la fausse amitié que Bill n'hésite pas à afficher jusqu'au moment de la confession. Une fois la vérité connue, le changement brusque de son comportement explique la lâcheté ou pire, le manque d'intérêt envers les personnes infectées :

Donc, depuis des mois, depuis l'aveu de ma maladie, Bill ne donnait plus aucun signe de vie, j'en souffrais parfois, cela augmentait parfois mon anxiété, ou mon regret de lui avoir parlé, mais, pour dire la vérité, son silence m'étonnait à peine, et je pourrais même ajouter que je m'en frottais les mains, car par ce silence abrupt, qui aurait pu sembler à quiconque une monstrueuse démission, Bill accédait cette fois au rang de personnage ambigu. (1990, p. 187)

L'image de Bill revient pour qu'elle disparaisse ensuite comme dans un cauchemar qui ne finit jamais. Ses promesses ne font que nourrir l'agonie de Guibert, qui lutte avec ses deux « je », l'un conscient du jeu trompeur de Bill, l'autre naïf et espérant accéder au moyen de guérir. Sauf les quelques pages du livre où la présence de Bill est à la fois déconcertante et nécessaire, l'écrivain n'insiste plus sur le rôle de sauveur de ce personnage. $\mathrm{Au}$ contraire, Guibert préfère adopter de nouveau son style direct et malicieux pour réaliser la description correcte de Bill :

Je jugeais l'attitude de Bill, sinon criminelle à mon endroit et si elle était réellement criminelle il va de soi tel que je suis que je m'en rengorgeais que davantage, inamicale au possible, et tout bêtement grossière. (1990, p. 196)

La dureté des mots couvre pourtant la tragédie de l'âme du malade, qui se trouve découvert devant le sida, qui ne lui laisse 
pas le temps d'attendre un miracle. Guibert n'oublie pas Bill et la possibilité de guérir que celui-ci lui enlève. L'amertume de la trahison disparaît à l'instant où le salut vient d'ailleurs : «j'attends avec impatience le vaccin littéraire qui me délivra du sortilège que je me suis infligé » (1990, p. 216). L'écriture sera le dernier et le plus fidèle compagnon dans le combat avec la mort et le sida, bien que cette métaphore de l'écriture/thérapie ne lui suffise pas dans sa démarche de terminer sa vie en même temps que ses livres.

Le sida ronge sans cesse et ne tient pas compte de la politique médicale que la France mène à la fin des années 1980. Guibert a de nouveau auprès de lui son ancien ami, Jules, qui lui procure le médicament DDI, le seul à l'aider à tenir debout. Le temps s'écoule chaque jour presque de la même manière: investigations et prises de sang, traitements commencés et arrêtés vite à cause des effets secondaires. L'affaiblissement du corps est de plus en plus ressenti par l'écrivain, qui veut à tout prix accomplir son projet. L'arrivée du DDI, médicament expérimenté sur les malades du sida aux États-Unis, revêt pour Guibert un rôle providentiel. Son existence tourne dès lors autour des efforts pour se fournir en DDI, devenu le lien avec la vie. Dans Le Protocole compassionnel, Guibert poursuit la description du sida, mais cette fois-ci, il s'agit de son sida. Auteur, narrateur et personnage, Guibert se déshabille devant les lecteurs et il le fait aux sens propre et figuré. Bien que Guibert soit contaminé depuis assez longtemps, il est à remarquer la difficulté pour l'écrivain d'accepter la maladie dans son corps :

Le lendemain du jour où le docteur Chandi m'annonçait cette décision de me faire arrêter l'AZT pour prendre du DDI, le docteur Nacier qui rédige désormais une gazette consacrée à la 
maladie (voyez comme j'ai du mal à prononcer le mot) m'envoyait le dernier numéro, dans lequel je trouvai ce gros titre: «290 malades morts aux États-Unis à la suite de la prescription de DDI. » (1991, p. 17)

Le désespoir d'approcher la mort éveille dans l'âme du malade les mêmes sentiments contradictoires auxquels il nous avait habitués. Toute nouvelle, bonne ou mauvaise, à propos d'un possible traitement du sida lui donne la force de continuer la bataille. Au-delà des aspects sociaux qui existent pleinement dans ses livres, l'épisode où Jules entre dans son appartement et dépose le sac avec «le DDI du danseur mort», phrase qui se répète d'une manière obsessive dans le livre, implique des connotations symboliques. D'abord, l'effet positif est perçu au niveau physique, quand la capacité de se mouvoir et de travailler de Guibert s'améliore :

Cela fait aujourd'hui cinq jours que je prends le médicament du mort, avant-hier je me suis senti un peu mieux dès le matin et j'ai entrepris ce récit qui, même s'il est sinistre, me semblait avoir une certaine gaieté, sinon vivacité, qui tient à la dynamique de l'écriture, et à tout ce qu'elle peut avoir d'imprévu. (1991, p. 19)

L'écriture est là, qui accompagne le DDI et qui en fait le couple parfait, parce que le couple qui se crée à l'instant est le seul capable à maintenir l'écrivain debout. La sève de la vie tirée de la disparition de l'autre représente la victoire de la vie sur la mort et la délivrance du mal.

À ce moment de notre analyse apparaît un autre couple de « je », celui du « je corps » et celui du « je âme». Le corps ne peut regagner ses forces que si l'âme ne se considère pas vaincue par la maladie. Ni vainqueur ni vaincu, Guibert se met à nu dans le dialogue direct avec ses lecteurs, à qui il laisse la liberté de juger son acte d'écriture : 
Aujourd'hui j'aimerais travailler sur une table de dissection. C'est mon âme que je dissèque à chaque nouveau jour de labeur qui m'est offert par le DDI du danseur mort. Sur elle je fais toute sorte d'examens, des clichés en coupe, des investigations par résonance magnétique, des endoscopies, des radiographies et des scanners dont je vous livre les clichés, afin que vous les déchiffriez sur la plaque lumineuse de votre sensibilité. (1991, p. 80)

L'auteur s'autoradiographie et devient ainsi l'équivalent d'un cliché médical prêt à être interprété. Ce qui étonne dans l'évolution de sa maladie et de son écriture, c'est la sensation de dépersonnalisation du «je » au fur et à mesure que Guibert avance dans son livre: "C'est le DDI du danseur mort, avec le Prozac, qui écrit mon livre, à ma place.» (1991, p. 84) Le sentiment d'être remplacé par le médicament agit comme un boumerang. La personnification du DDI annule la personnalité de l'écrivain. Conscient du danger de perdre son identité, Guibert prend la relève et reconquiert le « je » en reconnaissant les limites du DDI :

Je n'ai pas l'impression de ne plus être moi-même, ni d'être sorti de moi-même, ni d'être devenu un autre. Je suis le même qui pense pareil et qui l'écrit, auquel le médicament jusqu'à nouvel ordre donne l'énergie physique et morale de le faire. (1991, p. 84)

Guibert, malade qui a du mal à prononcer le mot "sida » et prenant conscience des limites du médicament, arrive maintenant à reconnaître aussi ses limites dans la guerre contre la maladie. Son livre se confond avec son corps et naît de la douleur et aussi du vide qui se crée à cause de la fin inévitable :

Et j'écris mon livre dans le vide, je le bâtis, le rééquilibre, pense à son rythme général et aux brisures de ses articulations, à ses ruptures et à ses continuités, à l'entremêlement de ses trames, à sa vivacité, j'écris mon livre sans papier ni stylo sous le chapiteau de la moustiquaire, jusqu'à l'oubli. (1991, p. 153) 
Écrire, jusqu'à l'épuisement, sur la maladie entraîne l'auteur dans un état d'oubli. Il veut oublier et surtout mépriser la mort à l'aide de mots sincères: "J'avais envie d'une écriture gaie, limpide, immédiatement communicante, pas d'une écriture tarabiscotée.» (1991, p.172) Cette affirmation, placée vers la fin du livre, porte en elle le message que Guibert a voulu transmettre à ses lecteurs et à la société. Son style, trop direct parfois, n'a fait que rendre la réalité du sida telle qu'il l'a vécue jusqu'au dernier moment de sa vie.

Chez Guibert, le sida est le « je » qui communique avec nous. Cette maladie irréversible prend la place de l'écrivain et dispose de sa personne à son gré. C'est au sida de dire quand le moment est venu de quitter le monde et de convoquer l'auteur malade à un duel qui le mènera à un dédoublement du «je ", celui du mourant et celui qui est en phase de rémission.

Philippe Hamon souligne que la description signifie "dévoiler, découvrir, ôter les masques, révéler, sonder, déchiffrer, lire, percer à jour, soulever le couvercle, démonter la machine, étudier les coulisses, mettre en lumière, aller au fond des choses » (1993, p. 62) : l'écrivain décrit pour trouver un sens à la réalité. Guibert a voulu trouver un sens à son existence déchirée entre le sida et l'homosexualité. Ainsi son corps est-il devenu le seul lien avec la vie et la description a-t-elle constitué un moyen de se trouver en tant qu'être humain. Si l'auteur y est parvenu, on ne saurait le dire, mais cette frange d'incertitude est indéniablement la marque de ses chefs-d'œuvre. 


\section{Bibliographie}

BlANCKEMAN, Bruno. (2000), Les Récits indécidables: Jean Echenoz, Hervé Guibert, Pascal Quignard, Lille, Presses universitaires du Septentrion.

BoulÉ, Jean-Pierre. (2001), Hervé Guibert: L'entreprise de l'écriture du moi, Paris, L'Harmattan.

Bruaire, Claude. (1968), Philosophie du corps, Paris, Seuil.

Buot, François. (1999), Hervé Guibert. Le jeune homme et la mort, Paris, Grasset.

GEnON, Arnaud. (2007), Hervé Guibert. Vers une esthétique postmoderne, Paris, L'Harmattan.

GuiBerT, Hervé. (1990), Á l'ami qui ne m'a pas sauvé la vie, Paris, Gallimard.

—. (1991), Le Protocole compassionnel, Paris, Gallimard.

HAMON, Philippe. (1993), Du descriptif, Paris, Hachette.

HERZlich, Claudine et Janine Pierret. (1991), Malades d'hier, malades d'aujourd'hui, Paris, Payot.

PigeAud, Jackie. (2009), Poésie du corps, Paris, Payot \& Rivages.

SARKONAK, Ralph (dir.). (1997), Le Corps textuel d'Hervé Guibert, Paris, Revue des Lettres Modernes.

SARTRE, Jean-Paul. (1960), L'Être et le Néant, Paris, Gallimard. 


\section{Résumé}

Entre espoir et désespoir, entre mort et guérison, Hervé Guibert ne cesse de se chercher et de se trouver. Nous analyserons dans cette étude le «je» malade et les caractéristiques de son esthétique au moment où la maladie ne peut plus être cachée et où l'écrivain décide de devenir le personnage principal de ses autofictions, Á l'ami qui ne m'a pas sauvé la vie et Le Protocole compassionnel. Faisant du sida une question personnelle, individuelle, l'écrivain n'a plus que son corps, qui est la seule liaison avec la vie. À travers l'analyse de son corps malade, Guibert peut se donner la certitude chaque matin, en se regardant dans le miroir, qu'il est encore vivant. Toute maladie, et d'autant plus le sida, agit d'abord sur le physique et ensuite sur le psychique du malade. Les changements évidents que le corps subit obligent la personne malade à se percevoir différemment à chaque étape de la maladie.

\section{Abstract}

Struggling between hope and despair, between death and recover, Hervé Guibert never gives up discovering himself. This study focuses on the inner sick person and on the aesthetics born as soon as the disease can't be hidden anymore. The writer decides to have AIDS as main character in his two novels, Á l'ami qui ne m'a pas sauvé la vie and Le Protocole compassionnel. Making it a personal issue, the body will be the only link to life. Through the deep analysis of his damaged body, Guibert assures himself of the fact that he is still alive. Considering the different stages of the disease, the physical and mental changes that occur make the patient see himself differently at every step of the way. 